\title{
Model of payment should not be the key concern when it comes to emergency department physicians' remuneration
}

\section{Howard Ovens, MD}

This month's CFEM includes a unique study on how changing a payment system affects emergency department (ED) flow and patient wait times. ${ }^{1}$ The authors strategically took advantage of a parting of the ways between two closely affiliated hospitals; one changed to fee for service (FFS), whereas the other remained on a pre-existing alternate contract. Conventional wisdom would suggest that, under FFS, flow would improve but the quality of care and perhaps even physician morale would suffer. Defenders of FFS-mostly physicians who prefer to practice this way, and their associations-would vehemently disagree. There is precious little evidence to resolve the dispute.

In this case, the authors found a very modest and non-sustained improvement in wait time but no change in the length of stay. What can we conclude from this? The authors review the issues and limitations of their study and rightly concede that their data cannot settle the debate. But is the classic debate-Is FFS better than alternative approaches to physician payment at ensuring flow and quality in the ED?-actually the right question? After 3 decades in ED administration and policy advocacy, I would say "No." Instead of searching for the ideal payment model, the more important consideration is the strategy that can be used to keep ED physicians happy in their jobs. The key factor in this is not how they are paid, but how much they are paid relative to their peers; this is where Canadian health systems are failing emergency medicine.

Experiences with alternate funding arrangements in Ontario over the past 2 decades have led to a majority of EDs opting for the contract option, with a small but stable minority remaining firmly in favour of FFS. ${ }^{2}$ The main differences between the two groups appear to be more philosophical than financial. The contract groups have a formal organization structure that creates group accountability with contractual protections ensuring fairness in decision-making and transparency around finances. An ED's annual volume and acuity determine gross funding, but the group can determine internal payment schemes, and there has been considerable innovation $^{3}$ in creating and adjusting these. Nothing prevents the FFS groups from pooling their income and emulating the same model in whole or in part on a voluntary basis. There is no doubt that some physicians will feel more comfortable in one sort of group culture than in another; although at a macro level in Ontario, distinguishing outcomes by a payment system is not possible.

To the extent that I am defending FFS, my comments are specific to emergency medicine. One of the major concerns about FFS in community practice is that it leads to a greater intensity of service and unnecessary visits as doctors fill their schedules with return visits. This does not apply to us in the ED; we have very little opportunity to control our volumes. I believe that choice and flexibility in payment systems are likely as or more important than details of the structure of a given system. I also believe that a group structure that includes collective responsibility over finances and scheduling can permit creative approaches to specific challenges of emergency medicine, such as coverage for unsocial shifts and concessions for seniority. I have observed groups stressed over non-financial approaches to covering nights, weekends, holidays, and so forth. While fairness and collegiality are critical, my own group has generally solved these problems painlessly by raising the relative value of hard-to-cover shifts until

From the ${ }^{*}$ Schwartz/Reisman Emergency Medicine Institute, Sinai Health System; and the ${ }^{\dagger}$ Department of Family and Community Medicine, University of Toronto, Toronto, ON

Correspondence to: Dr. Howard Ovens, 600 University Avenue, \#438, Toronto, ON M5G 1X5, Canada; Email: howard.ovens@ sinaihealthsystem.ca 
the problem is solved. If older doctors don't want to do nights, raise the value of a night shift until all are covered and let whoever wants to opt out of them pay the price in lower income or a higher number of other shifts.

However, the approach I suggested previously works in a fully staffed group only. This is not the norm; we have a large and growing deficit of emergency physicians in Ontario and Canada, as evidenced by recent surveys. ${ }^{4,5}$ The least productive emergency physician arrangement is the empty shift! A choice in payment scheme is an important factor in satisfaction and recruitment, as is the absolute level of remuneration. However, income relative to effort compared with competing disciplines may be the most important factor in creating an adequate and stable workforce. In emergency medicine, many practitioners are open to moving to a community practice in a traditional comprehensive primary care, or to alternatives such as urgent care or walk-in clinics. It is no accident to me that, in Ontario, and likely in most of the country, emergency medicine is chronically found to be low on objective relativity scales. Thus, my highest priority research question today would be: How does fee relativity for emergency medicine correlate with workforce adequacy? My hypothesis is that relativity would be the single greatest determinant. Because an adequate, stable, career-oriented workforce is a major factor in ensuring patient access to and quality of emergency care, relativity is likely far more important than any difference between FFS and alternate contracts.

My career as an emergency physician and chief of an ED has been fairly evenly divided between FFS and contract circumstances. We enjoyed a cohesive culture and managed to solve problems before and after we signed on to a contract. We actually voted against a contract the first two times that the option came up. We analysed the financials and decided that the contract did not offer an adequate premium to justify the bother in changing, until the third time around, when it did. I've been invited many times to business meetings of other Ontario ED groups facing this decision. The usual expectation is that I will be persuasive about the benefits of a contract over FFS, but I go over the nuances and pros and cons dispassionately and settle on one key question: Which approach in your circumstances will net the group the most money collectively? Then, take the money and decide how to use it to make your ED the best quality and happiest ED you can.

I am passionate about emergency medicine as a team sport and am dedicated to improving quality and safety in our EDs. Public interest is aligned with physician interest in creating and sustaining a discipline of adequate numbers of career-oriented and generally satisfied emergency physicians. A rigorous evaluation of public policy is crucial. But when it comes to evaluating payment schemes, my main question echoes that of Rod Tidwell in the 1996 movie Ferry Maguire: "Show me the money!"

Competing interests: None declared.

Keywords: emergency, physician compensation, fees, patient flow, quality, relativity, staffing, health human resources

\section{REFERENCES}

1. Innes GD, Scheuermeyer FX, Marsden J, et al. Impact of physician payment mechanism on emergency department operational performance. C7EM 2018;20(2):183-90, doi:10.1017/cem.2018.10.

2. Schull MJ, Vermeulen MJ. Ontario's alternate funding arrangements for emergency departments: the impact on the emergency physician workforce. CFEM 2005;7(2):100-6, doi:10.1017/S1481803500013051.

3. Whyne M, Whyne G, Rowe B. Variations in monetary distribution among Ontario's Alternative Funding Agreement workload model hospitals. CFEM 2007;9(1):21-5, doi:10.1017/S148180350001469X.

4. Collaborative Working Group on the Future of Emergency Medicine in Canada. Emergency medicine training of practice in Canada: celebrating the past of evolving for the future. Ottawa: The Canadian Association of Emergency Physicians, the College of Family Physicians of Canada, and the Royal College of Physicians and Surgeons of Canada; 2016.

5. Ontario LHIN lead survey of emergency directors, 2012 and 2015 - unpublished data. 